\title{
Criteria for the Convergence, Oscillation and Bistability of Pulse Circulation in a Ring of Excitable Media
}

\author{
H. SEDAGHAT ${ }^{1}$, C.M. KENT ${ }^{1}$, M.A. WOOD ${ }^{2}$
}

\begin{abstract}
A discrete model based on a nonlinear difference equation (equivalent to a coupled map lattice of high dimension) is used to study the dynamics of a circulating pulse in a ring of excitable media, such as cardiac cells. Based on the global and local properties of monotonic restitution and dispersion curves, criteria are obtained for the asymptotic stability of the unique steady state (pulse circulating at constant frequency) as well as for non-convergent oscillatory behavior of all non-equilibrium trajectories (pulse circulating at variable frequency). We also demonstrate that in certain cases the system is bistable, where an asymptotically stable equilibrium coexists with stable oscillatory solutions.
\end{abstract}

\section{Introduction.}

The periodic contractions of muscles that result in the beating of our hearts are caused by electrochemical signals or excitations called action potentials that propagate through chains of cardiac cells. Normally, cardiac cells generate and conduct action potentials in response to excitation by the self-oscillatory pacemaker cells in the heart's sinoatrial and atrioventricular nodes. However, in certain circumstances a closed loop or ring of tissue is formed within the heart that unidirectionally recycles a previously generated action potential. Such a reentrant circuit is capable of blocking the much slower pacemaker signals by transmitting its rapid pulses outward through adjacent cell layers, thus taking over the beating of the heart and leading to potentially life-threatening arrhythmias.

Reentry of an action potential pulse in a ring of cardiac cells or other excitable media and the resulting self-sustained propagation is relatively easy to model mathematically because of the simple one dimensional geometry. The study of such models contributes to our understanding of cardiac arrhythmias and the results of the study find concrete applications to experimental models of reentrant electrical activity in cardiac muscle. Nevertheless, the mathematical expressions of the manner in which a reentrant pulse propagates in a loop involve complex nonlinear equations whose study requires the application of a variety of different methods from the dynamical systems theory.

In Ito and Glass [14] a discrete model of a reentrant circuit is developed based on the restitution and dispersion properties of cardiac cells. Mathematically, the centerpiece of this model is a coupledmap lattice whose dimension is equal to the number of cells (or excitable units) in the loop. Close

\footnotetext{
${ }^{01}$ Department of Mathematics, Virginia Commonwealth University, Richmond, VA 23284-2014, USA;

${ }^{02}$ Department of Internal Medicine, Virginia Commonweath University Health Systems, Richmond, Virginia, 23298-0053, USA
} 
agreement was shown between certain important experimental facts and the model's simulations (using typical exponential-type maps to fit the restitution curve data). They discuss a number of issues, including the formation of a unidirectional block. For sustained propagation, they also study local stability and through numerical simulations establish the occurrence of discrete Hopf (or Neimark-Sacker) bifurcations with the variation of a parameter in their dispersion curve. In this way they exhibit the occurrence of almost periodic solutions with multiple incommensurate frequencies.

Two subsequent papers [5] and [6] by Courtemanche, Glass and Keener presented equivalent continuous space versions of the above model in terms of a delay differential equation and an integral delay equation, respectively. These papers present a mix of analytical and numerical results. The analytical results establish the occurrence of solutions with multiple frequencies via Hopf bifurcations in the continuous case. The length of the ring is used as the bifurcation parameter in these papers, where the non-convergent solutions arise when this length is sufficiently reduced. These issues are discussed in greater detail in [6] than in [5]. Notably, the numerical simulations of the delay-differential and the integral delay equation used different versions of the mapping in [14] for their discretizations. These versions are nearly equivalent and one of them is discussed in this paper.

The concepts of restitution and dispersion are well known and have been widely studied in various contexts, both theoretical and experimental; see, e.g., [2], [3], [5-7], [10], [13-16], [24-26]. In this paper we re-examine the dynamical system in [14], limiting our focus to dynamics of sustained reentry. Our purpose is two-fold: First, for sustained propagation our results extend those obtained in [14] to include global behavior in a bounded (but not infinitesimal) invariant region of the phase space. In particular, we obtain conditions for (a) the convergence of all orbits within the invariant region to a unique stable equilibrium so that the reentrant pulse circulates at constant-frequency; (b) the persistent oscillation (bounded but non-converging) of all non-equilibrium orbits, resulting in pulse circulation at variable frequency and (c) the occurrence of bistable behavior with coexisting stable oscillatory and convergent steady states so that it becomes possible to shift from one of these states to another through premature stimulations. Standard methods from the mathematical literature on discrete dynamics (e.g., [1], [8], [12], [17-20], [23]) are used to advantage in the proofs of Theorems 1 and 2 below.

Our second goal in this paper is to further generalize various results in previous studies by using generic restitution and dispersion curves that satisfy a few minimal conditions. This shows indirectly that the aforementioned qualitatively different types of behavior are general manifestations of the dynamical system under consideration and are not peculiar to a specific class of elementary mathematical functions. This substantial extension of the previously published material is made possible by our reliance on rigorous analytical methods. We use numerical simulations mostly in examples to illustrate the main results. 


\section{The Model.}

We consider a loop of cardiac tissue (or more generally, of excitable cells) of fixed length $L$ that consists of a fixed number $m$ of cells, or more generally, aggregate units or nodes in the sense of [14]. If the real number $\Delta L_{i}>0$ denotes the $i$-th cardiac unit spacing or inter-nodal separation, then $L=\sum_{i=1}^{m} \Delta L_{i}$. If we denote the average of $\Delta L_{i}, i=1,2, \ldots, m$ by $\Delta L$, and define

$$
\delta_{i}=\frac{\Delta L_{i}}{\Delta L}
$$

for each $i$, then

$$
\sum_{i=1}^{m} \delta_{i}=\sum_{i=1}^{m} \frac{\Delta L_{i}}{\Delta L}=m \quad \text { and } \quad L=m \Delta L .
$$

In most published works in the literature the spacings $\Delta L_{i}$ are assumed to deviate negligibly from the average $\Delta L$. In such cases one assumes that $\delta_{i}=1$ for all $i$ and considers a homogeneous loop in which all nodes are uniformly spaced. This is an important special case which captures all of the significant features with a minimum of technical details. Finally, for numerical simulations we arbitrarily take $m=500$, although much smaller values of $m$ give qualitatively similar outcomes $[14]$.

\subsection{Restitution curves.}

The APD Restitution. The action potential duration or APD is the length of time (usually measured in milliseconds or ms) that a node or cell is active after excitation by a pulse. After passage of the APD, if no new excitation takes place, the cell enters a recovery period called the diastolic interval or DI (also measured in $\mathrm{ms}$ ). The DI ends only by the arrival of a new excitation and it is only during the DI that a cell can fire or become active again if excited.

Let $A P D_{i, n}$ and $D I_{i, n}$ denote the APD and DI, respectively, for the cell $i$ in beat $n$. The most basic temporal relationship that is possible between the APD and the DI may be stated as

$$
A P D_{i, n}=A\left(D I_{i, n-1}\right), \quad i=1,2, \ldots, m
$$

where $A$ is an increasing single variable function whose graph is called the $A P D$-restitution curve.

We use the basic form (2) in this paper, but point out that in various papers (e.g., [4], [9], [11], [13], [15], [26]) it has been suggested that in general the function $A$ may contain additional delays or past APD dependence to account for memory effects.

Dispersion and the CT restitution. Each cardiac cell is capable of conducting the action potential pulse through it in a finite amount of time. The speed with which the pulse propagates through a cell is the conduction velocity or $\mathrm{CV}$, often measured in $\mathrm{cm} / \mathrm{sec}$. If $C V_{i, n}$ is the conduction velocity through cell $i$ in beat $n$ then we may express our second restitution hypothesis as follows:

$$
C V_{i, n}=V\left(D I_{i, n-1}\right), \quad i=1,2, \ldots, m
$$


where $V$ is a nondecreasing single variable function. Its graph is called the dispersion curve. With $\Delta L$ sufficiently small, we may assume that $V$ does not change from one end of a unit or cell to its other end so Eq.(3) defines a unit-wise or cellular conduction time restitution as follows

$$
C T_{i, n}=T\left(i, D I_{i, n-1}\right)=\frac{\Delta L_{i}}{V\left(D I_{i, n-1}\right)}=\frac{\delta_{i} \Delta L}{V\left(D I_{i, n-1}\right)}
$$

If we define the function of one variable

$$
C(t)=\frac{\Delta L}{V(t)}
$$

then $C T_{i, n}=\delta_{i} C\left(D I_{i, n-1}\right)$ for $i=1,2, \ldots, m$. Note that the function $C$ is nonincreasing; we refer to its graph as the $C T$-restitution curve. Except for the factor $\Delta L$ the function $C$ is the same as the "recovery curve" in [14].

\subsection{The propagation equation.}

During sustained reentry the pulse or the excitation front moves from node to node around the loop. The length of each cycle (or beat) $n$ is divided into two distinct periods, the APD followed by DI. The length of each cycle is also the sum of CT for all nodes or cells in the loop. Therefore, we have

$$
A P D_{i, n}+D I_{i, n}=\sum_{j=1}^{i-1} C T_{j, n+1}+\sum_{j=i}^{m} C T_{j, n}, \quad i=1,2, \ldots, m .
$$

Since in this setting $i=1$ is the reference point on the loop where a cycle begins and ends, the split in the sums reflects the fact that for $i>1$ conduction in cells 1 through $i-1$ takes place during beat $n+1$. Using the restitution relations, Eq.(4) may be written as the following system:

$$
D I_{i, n}=\sum_{j=1}^{i-1} T\left(j, D I_{j, n}\right)+\sum_{j=i}^{m} T\left(j, D I_{j, n-1}\right)-A\left(D I_{i, n-1}\right), i=1, \ldots, m .
$$

Eq.(5) is the coupled-map lattice discussed in [14]. It is also a partial difference equation in the spatial variable $i$ and the temporal variable $n$. See the paper by Stubna, Rand and Gilmour [24] for an adaptation of this argument to a non-loop structure.

Rather than working directly with Eq.(5), we first transform it to an ordinary $m$-th order difference equation by taking advantage of the periodic nature of the loop. Define the combined space-time variable

$$
x_{m n+i}=D I_{i, n}
$$

and note that

$$
A\left(D I_{i, n-1}\right)=A\left(x_{m(n-1)+i}\right)
$$


and

$$
\begin{aligned}
\sum_{j=1}^{i-1} T\left(j, D I_{j, n}\right)+\sum_{j=i}^{m} T\left(j, D I_{j, n-1}\right) & =\sum_{j=1}^{i-1} T\left(j, x_{m n+j}\right)+\sum_{j=i}^{m} T\left(j, x_{m(n-1)+j}\right) \\
& =\sum_{j=m n-m+i}^{m n+i-1} T\left(j, x_{j}\right)
\end{aligned}
$$

Next, we substitute $k=m n+i$ to get the following:

$$
x_{k}=\sum_{j=k-m}^{k-1} \delta_{j} C\left(x_{j}\right)-A\left(x_{k-m}\right)
$$

This equation is the main object of interest in this paper. It is equivalent to (5) provided that we extend the coefficient $\delta$ periodically, i.e., $\delta_{i+m n}=\delta_{i}$ for all positive integers $n$ and each $i=1,2, \ldots, m$ (a valid assumption since the loop consists of a finite number $m$ of units).

In the homogeneous case where $\delta_{i}=1$ for all $i$, Eq.(6) becomes an autonomous difference equation for which a greater number of results exist in the literature. This autonomous version of (6) is the same as that obtained in [6] upon a discretization of their independently developed integral equation (also see [7]). However, no analysis of the discrete case is given in these references.

Each solution of Eq.(6) is an infinite sequence $\left\{x_{k}\right\}_{k=1}^{\infty}$ of DI quantities that is generated by a given set of initial values which may be thought of as an initial state vector

$$
\mathbf{D I}_{0}=\left[D I_{1,0}, D I_{2,0}, \ldots, D I_{m, 0}\right]=\left[x_{-m+1}, x_{-m+2}, \ldots, x_{0}\right] .
$$

Also each subsequent state vector

$$
\mathbf{D I}_{n}=\left[x_{m(n-1)+1}, x_{m(n-1)+2}, \ldots, x_{m n}\right], \quad n \geq 0
$$

constitutes the dynamic state of the loop in one cycle. Each such vector determines the DI values for all the cells in the loop within a given cycle, and from the DI values and the restitution and dispersion functions, other quantities such as APD, CV, etc. can be computed. Further, plotting the components of $\mathbf{D I}_{n}$ as functions of the spatial coordinate $i$, one obtains the spatial profile of the DI values in each cycle (see Fig.2).

Exponential restitution and dispersion curves. Experimental data from pacing experiments as well as data generated by the numerical simulations of the ionic PDE models are typically fitted with exponential maps for numerical studies of pulse propagation. Monotonic maps that are variations of the following two functions appear frequently in the literature on cardiac electrophysiology:

$$
A(t)=a-b e^{-\sigma t}+p e^{-\gamma(t-\tau)^{2}}, \quad C(t)=\frac{\Delta L}{c}\left[1+d e^{-\omega t}\right]
$$


where the parameters $a, b, c, d, \tau, \sigma, \gamma, \omega>0$ and $p$ is a real number. We use these representations in our examples and figures below. The parameter $c$ is in fact the limiting (or maximum) value of conduction velocity; i.e., $c=\lim _{t \rightarrow \infty} V(t)$. The second exponential term has been added here to the definition of $A$ as a simple device for modifying $A$ locally near the value $\tau$. For small values of $|p|$ the mapping $A$ is strictly increasing and in this paper we shall be concerned with this range of $p$ values only. In particular, if $p=0$ then $A$ takes the form used in [14]; where the above definition of $A$ is used in the sequel (except for the one on bistability) we assume that $p=0$.

Using definitions (7) in Eq.(6) and re-arranging a few terms gives the following:

$$
x_{k}=\frac{d \Delta L}{c} \sum_{j=k-m}^{k-1} \delta_{j} e^{-\omega x_{j}}+b e^{-\sigma x_{k-m}}-p e^{-\gamma\left(x_{k-m}-\tau\right)^{2}}+\frac{L}{c}-a .
$$

Eq.(8) defines a complex dynamical system that displays a wide range of behaviors. Nevertheless, it is mathematically a rather special case of (6) in which certain situations do not occur that are possible for (6); e.g., nonconcavity or nondifferentiability. In this paper we generally use the following parameter values in (8):

$$
a=24, b=12, \sigma=0.5, \quad p=0, c=6, d=1, \omega=1, \Delta L=0.3 .
$$

unless otherwise stated. These values are largely arbitrary, but not far-fetched; they are within scientifically acceptable ranges and here they are used mainly for numerical verifications of our results in illustrative examples. For the sake of interpretation, $L=m \Delta L=150$ may be considered to be in mm (millimeters) and time units for DI, APD, etc. in various diagrams will be $10 \mathrm{~ms}$ (millisecond) each, unless otherwise specified.

\section{Dynamics of Sustained Reentry.}

A positive solution $\left\{x_{k}\right\}_{k=1}^{\infty}$ of (6), being an infinite sequence, represents sustained reentry. As noted above, a partitioning of this sequence into $m$-dimensional vectors gives the history of the loop's dynamic states in the phase space. In this section we study the qualitative properties of the solutions of $(6)$.

\subsection{The existence of a unique equilibrium.}

We begin with a set of basic assumptions concerning the restitution functions. The functions $A, C$ in (7) in particular satisfy all of these hypotheses.

(A1) There is $r_{A} \geq 0$ such that the APD restitution function $A$ is continuous and increasing on the interval $\left[r_{A}, \infty\right)$ with $A\left(r_{A}\right) \geq 0$. 
(A2) There is $r_{C} \geq 0$ such that the CT restitution function $C$ is continuous and nonincreasing on the interval $\left[r_{C}, \infty\right)$ with $\inf _{x \geq r_{C}} C(x) \geq 0$.

(A3) There is $r \geq \max \left\{r_{A}, r_{C}\right\}$ such that

$$
m C(r)>A(r)+r
$$

The first two assumptions express basic physiological facts that are commonly attributed to the functions $A$ and $C$. The third assumption guarantees (via Lemma 1 below) the existence of a structurally stable equilibrium or steady state. Physiologically, this means that conduction around the ring must be sufficiently slow to achieve the desired effect. Note also that (A1)-(A3) allow for the possibility that the restitution functions may not be monotonic or otherwise well-behaved near the origin.

Next, we define the auxiliary function $F=m C-A$ and note that by (A1)-(A3), $F$ is continuous and decreasing on the interval $[r, \infty)$ and satisfies

$$
F(r)>r
$$

Let $x^{*}$ be a steady state solution or equilibrium of (6), i.e., a solution of the equation

$$
x=\sum_{j=k-m}^{k-1} \delta_{j} C(x)-A(x)=m C(x)-A(x)=F(x) .
$$

In particular, $x^{*}$ is also a fixed point of the auxiliary map $F$ so it is the same in the autonomous case $\delta_{i}=1$.

Lemma 1. Assume that (A1)-(A3) hold.

(a) Eq.(6) has a unique positive equilibrium $x^{*} \in(r, F(r))$.

(b) $\left(x^{*}, F(r)\right]=F\left(\left[r, x^{*}\right)\right)$.

(c) $[r, F(r)]=\left[r, x^{*}\right] \cup F\left(\left[r, x^{*}\right)\right)$ disjointly.

Proof. (a) Let $f(x)=F(x)-x$ so that by (11) $x^{*}$ is a zero of $f$. Then by (10) $f(r)>0$. Further, since $F$ is decreasing for $x \geq r$, applying $F$ to (10) gives

$$
f(F(r))=F(F(r))-F(r)<0 .
$$

The existence of $x^{*} \in(r, F(r))$ is now established by applying the Intermediate Value Theorem to the continuous $f$. The uniqueness of $x^{*}$ is a clear consequence of the strictly decreasing nature of $f$.

(b) Let $y \in\left(x^{*}, F(r)\right]$. Then $x=F^{-1}(y)>F^{-1}(F(r))=r$ and $x<F^{-1}\left(x^{*}\right)=x^{*}$. Thus $\left(x^{*}, F(r)\right] \subset F\left(\left[r, x^{*}\right)\right)$. The converse is clear. 
(c) The disjoint union follows immediately from Part (b).

The interval $[r, F(r)]$ represents a relevant interval for this model since what happens outside this interval is not relevant to our discussion of sustained reentry. The relevant interval is not generally invariant, though it may contain an invariant interval.

Remark. We are interested only in non-negative DI values; therefore, if a zero of $F$ occurs in the relevant interval, i.e., if there is $p \in[r, F(r)]$ such that $F(p)=0$ then $F(x)<0$ for $x>p$ so the relevant interval reduces to $[r, p]$. We note that since $F\left(x^{*}\right)=x^{*}>0$, it must be that $x^{*} \in(r, p)$.

\subsection{The invariant interval.}

The existence of a nontrivial invariant interval in particular guarantees an open set of bounded solutions for (6) and thus assures the robust occurrence of sustained reentry. An additional hypothesis is required.

(A4) There is $s \in\left[r, x^{*}\right)$ such that $s \leq F(F(s))=F^{2}(s)$.

Lemma 2. Assuming (A1)-(A4), the interval $I=[s, F(s)]$ is nontrivial, contains $x^{*}$ and is invariant for Eq.(6); i.e., if the initial values $x_{0}, \ldots, x_{-m+1}$ are in $I$, then $x_{k} \in I$ for all $k \geq 1$. Also $I \subset[r, F(r)]$.

Proof. First, observe that since $s<x^{*}$ and $F$ is decreasing, then $F(s)>x^{*}>s$. Hence, the interval $I$ contains $x^{*}$ and is nontrivial, i.e., it has a nonempty interior. Next, assume that $x_{0}, \ldots, x_{-m+1} \in I$. Then for $j=-m+1, \ldots, 0, x_{j} \geq s$ so $A\left(x_{-m+1}\right) \geq A(s)$ and $C\left(x_{j}\right) \leq C(s)$. Therefore,

$$
x_{1}=\sum_{j=-m+1}^{0} \delta_{j} C\left(x_{j}\right)-A\left(x_{-m+1}\right) \leq m C(s)-A(s)=F(s) .
$$

Similarly, for $j=-m+1, \ldots, 0, x_{j} \leq F(s)$ so $A\left(x_{-m+1}\right) \leq A(F(s))$ and $C\left(x_{j}\right) \geq C(F(s))$. Therefore,

$$
\begin{aligned}
x_{1} & =\sum_{j=-m+1}^{0} \delta_{j} C\left(x_{j}\right)-A\left(x_{-m+1}\right) \\
& \geq m C(F(s))-A(F(s)) \\
& =F(F(s)) \\
& \geq s .
\end{aligned}
$$

It follows that $x_{1} \in I$. Now, assume inductively that for $k \geq 1$, we have established that $x_{k-1}, \ldots, x_{k-m} \in I$. Then repeating the above argument gives $x_{k} \in I$ and shows $I$ to be invariant. 


\subsection{Convergence to a stable steady state.}

In this section we look at a special case of (A4) that implies the asymptotic stability of the equilibrium (convergence of all trajectories in $I$ to a stable steady state). This special case of condition (A4) is stated as follows:

(A4S) There is $s \in\left[r, x^{*}\right)$ such that $F^{2}(x)>x$ for all $x \in\left(s, x^{*}\right)$.

Figure 1 depicts a case where (A4S) holds with $r=s=0$. Note in particular that if $A^{\prime}\left(x^{*}\right)<1$ then it is easy to see that (A4S) holds, at least when $C^{\prime}\left(x^{*}\right)=0$ (though $C$ need not be constant). However, (A4S) is a weaker condition in that the differentiability of $A$ is not required and that if differentiable, then the derivative $A^{\prime}$ need not be uniformly bounded by 1 in a left-neighborhood of $x^{*}$ (i.e., small irregularities in the APD curve do not affect the qualitative behavior of the circulating pulse).

Before presenting Theorem 1, it is necessary to consider the dynamics of the auxiliary map $F$ under (A4S). It is known [23, Sec.2.1] that condition (A4S) is in fact necessary and sufficient for $x^{*}$ to attract all orbits of $F$ that start in $(s, F(s))$. But since $F$ is a relatively simple mapping, we can be more specific here.

Lemma 3. Assume that (A1)-(A3) plus (A4S) hold. Then for every $x_{0} \in\left(s, x^{*}\right)$

$$
s<x_{0}<F^{2}\left(x_{0}\right)<\cdots<x^{*}<\cdots<F^{3}\left(x_{0}\right)<F\left(x_{0}\right)<F(s)
$$

and

$$
\lim _{n \rightarrow \infty} F^{2 k}\left(x_{0}\right)=\lim _{n \rightarrow \infty} F^{2 k+1}\left(x_{0}\right)=x^{*} .
$$

Proof. Since $F$ is decreasing, if $x_{0} \in\left(s, x^{*}\right)$ then $F\left(x_{0}\right)>F\left(x^{*}\right)=x^{*}$ and $F\left(x_{0}\right)<F(s)$. Thus

$$
x^{*}<F\left(x_{0}\right)<F(s) .
$$

Applying $F$ to (14) in the above fashion gives

$$
F^{2}(s)<F^{2}\left(x_{0}\right)<x^{*} .
$$

Now (12) follows by simple induction. Statements (13) follow from (12) because $F$ has no fixed points in $(s, F(s))$ other than $x^{*}$ to which the odd and even iterates of $F$ can converge.

Theorem 1. Let (A1)-(A3) and (A4S) hold. Then the equilibrium $x^{*}$ is stable and every solution of (6) with initial values in $(s, F(s))$ is attracted to $x^{*}$.

Proof. First we establish the attracting nature of $x^{*}$. Let $x_{0}, \ldots, x_{-m+1}$ be in the interval $(s, F(s))$, and define

$$
\mu_{1}=\min \left\{x^{*}, x_{0}, \ldots, x_{-m+1}\right\}, \quad \mu_{2}=\max \left\{x^{*}, x_{0}, \ldots, x_{-m+1}\right\} .
$$


Since $F$ is continuous, we have $F(x) \rightarrow F(s)$ as $x \rightarrow s$. Thus we can find $q \in\left(s, \mu_{1}\right)$ sufficiently close to $s$ that $F(q) \in\left(\mu_{2}, F(s)\right)$. Next, observe that since $x_{0}, \ldots, x_{-m+1}>q$,

$$
x_{1}=\sum_{j=-m+1}^{0} \delta_{j} C\left(x_{j}\right)-A\left(x_{-m+1}\right)<m C(q)-A(q)=F(q)
$$

Similarly, $x_{0}, \ldots, x_{-m+1}<F(q)$ implies

$$
x_{1}=\sum_{j=-m+1}^{0} \delta_{j} C\left(x_{j}\right)-A\left(x_{-m+1}\right)>m C(F(q))-A(F(q))=F^{2}(q) .
$$

If (A4S) holds, then $F^{2}(q)>q$ so that $x_{1} \in\left(F^{2}(q), F(q)\right) \subset(q, F(q))$. Repeating a similar calculation for $x_{2}, \ldots, x_{m}$ we conclude that

$$
x_{k} \in\left(F^{2}(q), F(q)\right) \subset(q, F(q)), \quad k=1, \ldots, m .
$$

Next, we move on to the next cycle and look at $x_{m+1}$. Since by $(15) x_{1}, \ldots, x_{m}>F^{2}(q)$,

$$
x_{m+1}=\sum_{j=1}^{m} \delta_{j} C\left(x_{j}\right)-A\left(x_{1}\right)<m C\left(F^{2}(q)\right)-A\left(F^{2}(q)\right)=F^{3}(q) ;
$$

further, $x_{1}, \ldots, x_{m}<F(q)$ gives

$$
x_{m+1}=\sum_{j=1}^{m} \delta_{j} C\left(x_{j}\right)-A\left(x_{1}\right)>m C(F(q))-A(F(q))=F^{2}(q) .
$$

Since $F^{3}(q)<F(q)$, this argument can be repeated for $x_{m+2}, \ldots, x_{2 m}$ to yield

$$
x_{k} \in\left(F^{2}(q), F^{3}(q)\right) \subset\left(F^{2}(q), F(q)\right), \quad k=m+1, \ldots, 2 m .
$$

Continuing this argument inductively leads to the conclusion that

$$
\begin{array}{ll}
x_{k} \in\left(F^{2 n}(q), F^{2 n-1}(q)\right), & k=m(2 n-2)+1, \ldots, m(2 n-1) \\
x_{k} \in\left(F^{2 n}(q), F^{2 n+1}(q)\right), & k=m(2 n-1)+1, \ldots, 2 m n .
\end{array}
$$

From these relations and Lemma 3 it is clear that $x_{k}$ converges to $x^{*}$ as $k \rightarrow \infty$. It remains to show that $x^{*}$ is stable (dynamically in the sense of Liapunov). Let $\varepsilon>0$ and use the continuity of $F$ to pick $\delta \in(0, \varepsilon)$ small enough that $F\left(x^{*}-\delta\right)<x^{*}+\varepsilon$. If $x_{0}, \ldots, x_{-m+1} \in\left(x^{*}-\delta, x^{*}+\delta\right)$ then it follows from Lemma 3 and (16) that

$$
x_{k} \in\left(x^{*}-\delta, F\left(x^{*}-\delta\right)\right) \subset\left(x^{*}-\varepsilon, x^{*}+\varepsilon\right), \quad k \geq 1 .
$$


Hence $x^{*}$ is stable.

\section{Remarks.}

1. If $x^{*}$ is attracting (e.g., conditions of Theorem 1 hold) then the cycle length $m C\left(x^{*}\right)$ may be easily computed as the fixed period $T^{*}$ (analogous to the basic cycle length or BCL) for the oscillation of the reentrant pulse. Note that

$$
T^{*}=m C\left(x^{*}\right)=\frac{m \Delta L}{V\left(x^{*}\right)}=\frac{L}{V^{*}}
$$

where $V^{*}$ is steady state conduction velocity in the loop. Similarly, the APD is calculated from the restitution relation as $A^{*}=A\left(x^{*}\right)$. It may be emphasized that since $F\left(x^{*}\right)=x^{*}$ we have the cycle period $T^{*}=A^{*}+x^{*}>A^{*}$ as required.

The frequency $1 / T^{*}$ can be quite high in this sort of reentrant regime. We calculate this frequency in a hypothetical case using Eq.(8) subject to the parameter values (9) but with $L$ increased to 162 (from 150 by increasing $m$ to 540). In this case, we obtain the situation depicted in Fig. 1 so by Theorem 1 the fixed point $x^{*} \approx 4.55$ (estimated numerically and interpreted as a steady state DI of $45.5 \mathrm{~ms}$ ) is globally asymptotically stable (with respect to $I$ ).

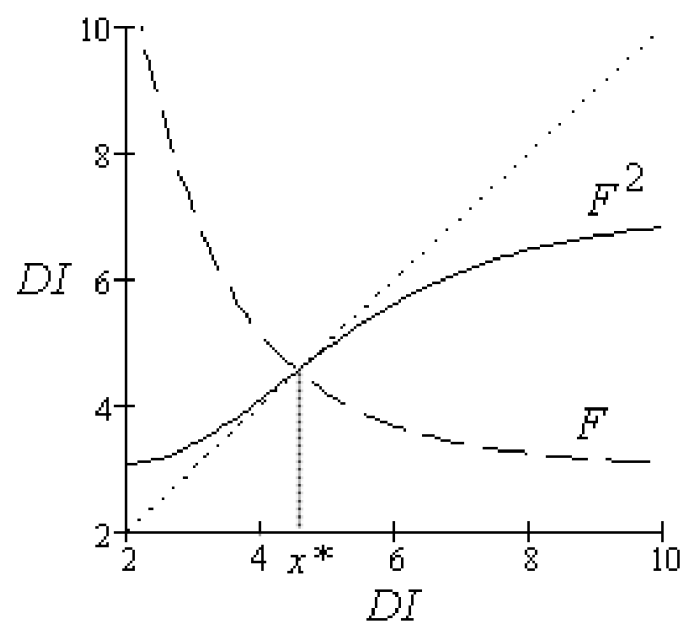

Figure 1: Attracting Equilibrium

FIG.1: This figure illustrates conditions for the stability of the fixed point $x^{*}$ of the mapping $F$, which is required in Theorem 1. When the graph of $F^{2}=F \circ F$ crosses the diagonal going from above it to below it as shown, $x^{*}$ is asymptotically stable. 
The fixed cycle length or period of the reentrant pulse is approximately

$$
m C(4.55)=\frac{L}{c}\left(1+d e^{-4.55 \omega}\right)=\frac{162}{6}\left(1+e^{-4.55}\right)=27.3
$$

which we interpret as 273 milliseconds, corresponding to a frequency of $60000 / 273$ or a rather fast 220 cycles (or beats) per minute.

2. (A1)-(A3) plus (A4S) are sufficient for the asymptotic stability of the equilibrium but in general they are not necessary. A special case where these hypotheses are both necessary and sufficient for asymptotic stability is when $C$ is constant (see Theorem 2.1.2 in [23]).

\subsection{Persistent oscillations.}

In a series of experiments on animal cardiac tissue by Frame and Simson [10] it was found that the reentrant pulse does not always cycle around a loop with a fixed period. In some cases, the cycle length tended to oscillate without approaching a specific value. In [14] these oscillations were attributed to the appearance of quasiperiodic solutions for (5) due to local bifurcations (NeimarkSacker or discrete Hopf). In this section we discuss sufficient conditions for oscillations to occur in all nontrivial solutions of (6) within the invariant $I^{m}$. Throughout this section we assume that the restitution functions $A$ and $C$ are continuously differentiable and that $\delta_{i}=1$ for all $i$, i.e., the loop is homogeneous.

For the autonomous version of (6), the characteristic polynomial of the linearization at $x^{*}$ is given as

$$
P(\lambda)=\lambda^{m}+\sum_{i=1}^{m-1} \beta \lambda^{i}+\beta+\alpha, \quad \alpha=A^{\prime}\left(x^{*}\right)>0, \beta=-C^{\prime}\left(x^{*}\right) \geq 0 .
$$

See e.g., [18] or [23]. Note that the roots of $P$ are the eigenvalues of the linearization of (6) at $x^{*}$. We now list some special properties of $P$.

Lemma 4. (a) $P$ has no non-negative (real) roots.

(b) If some root of $P$ lies on the unit circle in the complex plane, then $\alpha+\beta=1$;

(c) If $\alpha+\beta=1$ then for each root $\lambda$ of $P, 1 / \lambda$ is also a root.

(d) If $\beta=0$ (even if $C$ is not constant) then either all roots of $P$ are inside the unit disk in the complex plane if $\alpha<1$ or they are all outside if $\alpha>1$.

Proof. (a) This is clear from the facts that $\beta \geq 0$ and $\alpha+\beta>0$.

(b) First we show that (i) implies (iii). Let $\alpha+\beta=1$. By Part (b), $\operatorname{roots} \lambda_{j}=\rho_{j} \exp \left(i \theta_{j}\right)$ of $P$ must have modulus $\rho_{j}=1$ for all $j=1, \ldots, m$ and thus they are on the unit circle. In particular, the only possible real root of $P$ is -1 which occurs when $m$ is odd.

Next, (iii) trivially implies (ii), so it remains to show that (ii) implies (i). Let $\lambda_{1}$ be a root that is on the unit circle. Then $\lambda_{1}=\exp \left(i \theta_{1}\right)$ so the conjugate $\exp \left(-i \theta_{1}\right)=1 / \lambda_{1}$ is also a root. Since 
$P\left(\lambda_{1}\right)=0$ it follows that

$$
\beta \sum_{i=1}^{m-1} \lambda_{1}^{i}=-\lambda_{1}^{m}-\alpha-\beta
$$

Also $P\left(1 / \lambda_{1}\right)=0$ so

$$
\begin{aligned}
0 & =\frac{1}{\lambda_{1}^{m}}+\beta \sum_{i=1}^{m-1} \frac{1}{\lambda_{1}^{i}}+\beta+\alpha \\
& =\frac{1}{\lambda_{1}^{m}}\left[1+\beta \sum_{i=1}^{m-1} \lambda_{1}^{i}\right]+\alpha+\beta \\
& =\frac{1-\lambda_{1}^{m}-\alpha-\beta}{\lambda_{1}^{m}}+\alpha+\beta \\
& =\left(\frac{1}{\lambda_{1}^{m}}-1\right)(1-\alpha-\beta)
\end{aligned}
$$

Note that $\lambda_{1}^{m} \neq 1$, since otherwise the sum of the $m$-th roots of unity in (17) would add up to -1 , leaving $-\beta$ on the left but giving $-1-\alpha-\beta$ on the right. Therefore, by (18) it is the case that $1-\alpha-\beta=0$ as required.

(c) If $\alpha+\beta=1$ then

$$
P\left(\frac{1}{\lambda}\right)=\frac{1}{\lambda^{m}}+\beta \sum_{i=1}^{m-1} \frac{1}{\lambda^{i}}+1=\frac{1}{\lambda^{m}}\left[\lambda^{m}+\beta \sum_{i=1}^{m-1} \lambda^{i}+1\right]=\frac{P(\lambda)}{\lambda^{m}} .
$$

(d) This is straightforward since $P$ reduces to the simple equation $\lambda^{m}+\alpha$ if $\beta=0$.

Next, a global oscillation result [23, p.166] is needed which we quote here as a lemma. We say that a sequence $\left\{x_{n}\right\}_{n=1}^{\infty}$ oscillates persistently if it is bounded and has at least two distinct limit points. In particular, persistently oscillating solutions cannot converge to a point.

Lemma 5. Consider the general difference equation

$$
x_{n}=f\left(x_{n-1}, \ldots, x_{n-m}\right)
$$

where $f: D \rightarrow \mathbb{R}$ for a set $D \subset \mathbb{R}^{m}$. Assume that (19) has a unique fixed point $x^{*}$ and that all the eigenvalues of the linearization of (19) at $x^{*}$ (i.e., the roots of its characteristic polynomial) lie outside the unit disk in the complex plane. Further, assume that

$$
f\left(x^{*}, \ldots, x^{*}, x\right) \neq x^{*} \quad \text { if } \quad x \neq x^{*} .
$$

Then all nontrivial solutions of (19) that are bounded oscillate persistently.

We are now ready for the main result of this subsection. 
Theorem 2. Assume that (A1)-(A4) and one of the following inequalities hold:

$$
A^{\prime}\left(x^{*}\right)+(m-2) C^{\prime}\left(x^{*}\right)>1
$$

or

$$
A^{\prime}\left(x^{*}\right)>0, \quad C^{\prime}\left(x^{*}\right) \leq-1 .
$$

Then $x^{*}$ is unstable and all nontrivial solutions of (6) oscillate persistently in the invariant interval $I$.

Proof. In the notation of Lemma 4, Inequality (21) may be written as

$$
\alpha-(m-2) \beta>1 \text {. }
$$

We first show that if (23) holds then all roots of the characteristic polynomial $P$ lie outside the unit disk in the complex plane. To this end, define the polynomial

$$
Q(\lambda)=\frac{\lambda^{m}}{\alpha+\beta} P\left(\frac{1}{\lambda}\right)=\lambda^{m}+\frac{1}{\alpha+\beta} \sum_{j=1}^{m-1} \beta \lambda^{j}+\frac{1}{\alpha+\beta} .
$$

and observe that $\lambda_{0}$ is a root of $Q$ if and only if $1 / \lambda_{0}$ is a root of $P$. Thus if every root of $Q$ is inside the unit disk, then every root of $P$ will be outside as desired. A well-known sufficient condition (e.g., $[23$, pp.209,210]) for all the roots of $Q$ to be inside the unit disk is that the sum of all the coefficients (except for that of the highest power $\lambda^{m}$ ) be less than unity, i.e.,

$$
1>\sum_{j=1}^{m-1} \frac{\beta}{\alpha+\beta}+\frac{1}{\alpha+\beta}=\frac{(m-1) \beta+1}{\alpha+\beta} .
$$

It is easy to see that (24) is equivalent to (23).

Next, assume that (22) holds. Then in the notation of Lemma $4, \beta \geq 1$ with $\alpha>0$; in particular, $\alpha+\beta>1$. If all roots of $P$ are not outside the unit disk, then some root

$$
\lambda_{0}=r_{0} e^{i \theta_{0}}=r_{0}\left(\cos \theta_{0}+i \sin \theta_{0}\right)
$$

of $P$ has modulus $r_{0} \leq 1$. By Lemma 4(c) we may assume that $r_{0}<1$. Setting $P(\lambda)=0$ and writing its middle terms in a compact way gives the equation

$$
\lambda^{m}+\beta \frac{\lambda^{m}-1}{\lambda-1}+\alpha=0
$$

Since by Part (a) $\lambda \neq 1$ for any root, the zeros of (25) are precisely those of $P(\lambda)=0$ and also the same as the non-unit zeros of

$$
\lambda^{m}(\lambda-1)+\beta\left(\lambda^{m}-1\right)+\alpha(\lambda-1)=0 .
$$


Inserting $\lambda_{0}$ in (26) and re-arranging terms, we get

$$
\lambda_{0}^{m+1}+(\beta-1) \lambda_{0}^{m}+\alpha \lambda_{0}=\alpha+\beta .
$$

Setting the real parts on the two sides of (27) equal we obtain:

$$
r_{0}^{m+1} \cos (m+1) \theta_{0}+(\beta-1) r_{0}^{m} \cos m \theta_{0}+\alpha r_{0} \cos \theta_{0}=\alpha+\beta .
$$

For $\beta \geq 1$ and $\alpha>0$ the left side of (28) is bounded above by the quantity

$$
r_{0}^{m+1}+(\beta-1) r_{0}^{m}+\alpha r_{0}<1+\beta-1+\alpha=\alpha+\beta .
$$

But this contradicts equation (28) which was assumed to hold with $r_{0} \in(0,1)$ for some $\theta_{0}$. It follows that $\lambda_{0}$ cannot exist and thus all roots of $P$ must be outside the unit circle.

Having shown that a unique, unstable equilibrium exists in a positively invariant region, the proof can now be concluded by Lemma 5 as soon as we show that (20) holds. For Eq.(6), the function $f$ is given by

$$
f\left(u_{1}, \ldots, u_{m}\right)=\sum_{j=1}^{m} C\left(u_{j}\right)-A\left(u_{m}\right)
$$

so (20) is equivalent to

$$
(m-1) C\left(x^{*}\right)+C(x)-A(x) \neq x^{*} \quad \text { if } \quad x \neq x^{*} .
$$

Since $x^{*}=F\left(x^{*}\right)=m C\left(x^{*}\right)-A\left(x^{*}\right),(29)$ is equivalent to

$$
C(x)-A(x) \neq C\left(x^{*}\right)-A\left(x^{*}\right) \quad \text { if } \quad x \neq x^{*}
$$

This last inequality is true since the function $C(x)-A(x)$ is strictly decreasing on the invariant interval $I$.

\section{Remarks.}

1. Writing (21) in the equivalent form

$$
A^{\prime}\left(x^{*}\right)>1+(m-2)\left|C^{\prime}\left(x^{*}\right)\right|
$$

we see that for any given value of $A^{\prime}\left(x^{*}\right)$ larger than 1 , persistent oscillations occur by Theorem 2 if $\left|C^{\prime}\left(x^{*}\right)\right|$ is sufficiently small, i.e., if $C$ is sufficiently flat in a neighborhood of $x^{*}$. On the other hand, if $A^{\prime}\left(x^{*}\right)<1$ then the steepness condition $\left|C^{\prime}\left(x^{*}\right)\right| \geq 1$ is sufficient to guarantee the occurrence of persistent oscillations. Thus in this sense (21) and (22) are complementary conditions.

As an application of Theorem 2, consider Eq.(8) subject to the parameter values (9) except with $\omega=2$ now. Then we may estimate $x^{*} \approx 3.32$ (DI of about $33.2 \mathrm{~ms}$ ) and compute

$$
A^{\prime}\left(x^{*}\right) \approx 1.141, \quad 1+(m-2)\left|C^{\prime}\left(x^{*}\right)\right| \approx 1.065 .
$$


Thus (21) is satisfied and solutions oscillate by Theorem 2. Fig.2 shows both the spatial and temporal DI profile in six consecutive cycles of a sample trajectory (the thick curve) after the transient effects have dissipated; in addition to its oscillatory nature, this solution is quasiperiodic, a fact that does not follow from Theorem 2 but may be inferred from local analysis.

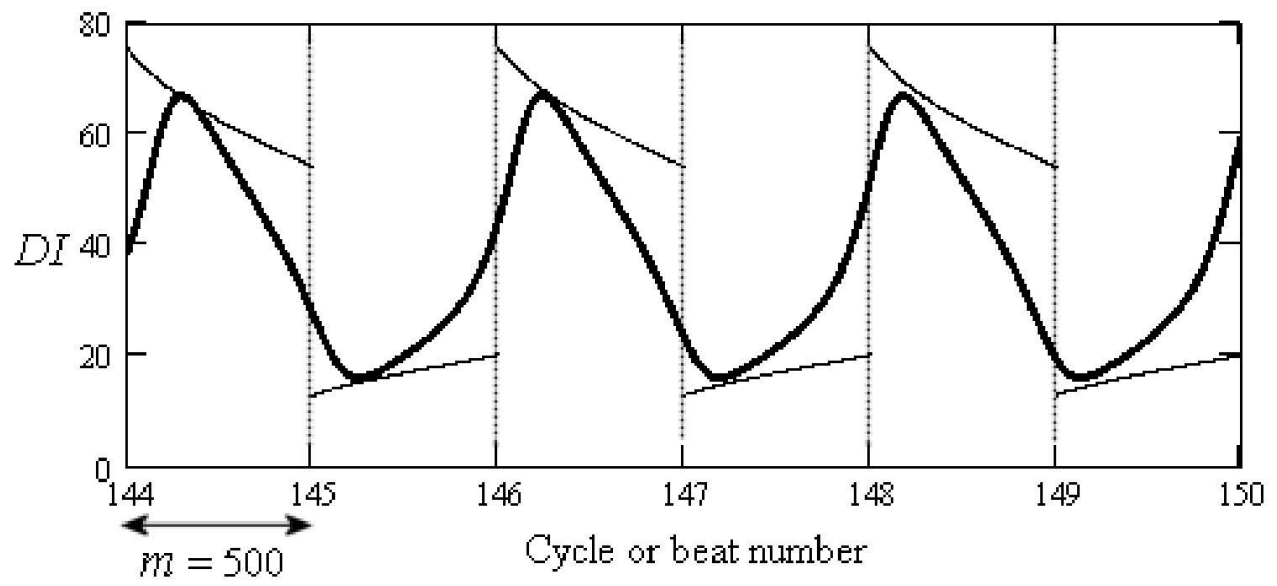

Figure 2: Different types of oscillations

FIG.2: Two different types of oscillatory solutions: The thick curve shows quasiperiodic oscillations when $\omega=2$ and the thin curves show periodic oscillations when $\omega=3$. Each point on either curve indicates the DI value of a particular unit in a given cycle. Both of the curves shown are generated from the same initial configuration vector $D I_{0}=[c, \ldots, c]$ where $c$ is a positive constant that may represent, e.g., the dynamic state of the loop in terms of cellular DI values just before the initiation of reentry. In each of the vertial strips each curve indicates the configuration of DI values over the entire ring (500 units) in one cycle or beat.

Alternatively, if we have a CT restitution curve that flattens more quickly (e.g., $\omega=3$ ), then we would get the solution shown by the thin curves in Fig.2. In this case, Theorem 2 again confirms that this solution is oscillatory because

$$
x^{*} \approx 3.29, \quad A^{\prime}\left(x^{*}\right) \approx 1.158, \quad 1+(m-2)\left|C^{\prime}\left(x^{*}\right)\right| \approx 1.004 .
$$

We note in passing that although $A$ is independent of $\omega$, if $C$ is not constant then changes in $\omega$ affect $x^{*}$ and thus the value of the slope $A^{\prime}\left(x^{*}\right)$.

2. In [14] it is conjectured that $x^{*}$ is locally asymptotically stable (or unstable) if the quantity $A^{\prime}\left(x^{*}\right)-C^{\prime}\left(x^{*}\right)-1$ is negative (respectively, positive). Although neither (21) nor (22) implies the positivity of this quantity, numerical simulations and certain results such as Lemma 4 suggest that 
this conjecture is probably true. In the notation of this paper, we restate this open problem as follows: All roots of the characteristic polynomial $P$ are inside (respectively, outside) the unit disk if and only if $\alpha+\beta<1$ (respectively, $\alpha+\beta>1$ ).

\subsection{Bistability.}

When cardiac tissue receives a premature stimulus such as an electrical shock of the type imparted by a defibrillator or through electrodes in a lab preparation, it can change its rhythm. In the case of a one dimensional reentrant circuit, a particular type of behavior such as stably convergent may change to stably persistent oscillatory or conversely. Such changes can occur if Eq.(6) is bistable (or more generally, multistable; note that a premature stimulus in cycle $n$ changes the components of $\mathbf{D I}_{n}$, thus shifting the orbit in phase space). In this section we use Theorems 1 and 2 to show that a type of bistability that is caused by slight "dents" in the APD curve may exist in a general setting. An advantage of this approach is that the emergence of bistability in the higher order equation (6) can be observed in a bifurcation diagram of the one dimensional auxiliary map $F$ even when $C$ is not constant; see the Remark below.

The main idea: Consider a case where the solutions of (6) are persistently oscillatory, e.g., inequality (21) is satisfied at the equilibrium under hypotheses (A1)-(A4). Suppose that now the $A P D$ curve is made locally flat (i.e., its slope small) in the vicinity of the unstable equilibrium so that condition $(A 4 S)$ holds in an interval $I_{0}$ containing $x^{*}$ ( $A$ is unaltered or altered negligibly outside $I_{0}$ ). The endpoints of $I_{0}$ then represent an unstable 2-cycle for the auxiliary map $F$. Further, assume that $I_{0}$ is small enough to not contain some previously oscillatory solutions (in the sense that the oscillatory orbit in the phase space does not enter the hypercube $I_{0}^{m}$ ). Then by Theorem 1 solutions that are generated by initial values inside $I_{0}$ will converge to a new stable equilibrium without substantially affecting the previously oscillatory solutions that were outside $I_{0}$. The resulting system is thus bistable.

We emphasize that the existence of $I_{0}$ is sufficient but not necessary for the bistability of (6), unless of course, the CT restitution curve $C$ is constant. Let us now illustrate the preceding ideas using Eq.(8) subject to the parameter values (9) except that we now set $p=0.3$ (with $\gamma=1$, $\tau=2.5$ ) in order to modify the APD curve, and also pick a suitable value for $\omega$ to enhance the bistability effect. The graph of $F^{2}(t)-t$ in Fig. 3 shows the primary invariant interval $I$ discussed previously, as well as the smaller one $I_{0}$ for a particular value of $\omega$. 


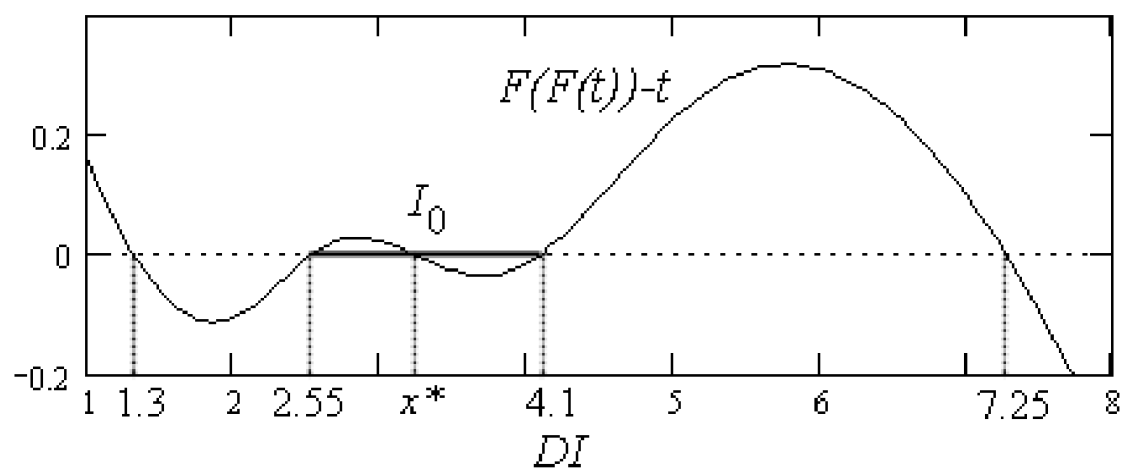

Figure 3: Two invariant intervals

FIG.3: The two invariant intervals $I=[1.3,7.25]$ and $I_{0}=[2.55,4.1]$ with the latter highlighted as the interval of convergence.

Figure 4 below shows the different outcomes that result depending on whether we choose the initial values in $I_{0}$ or outside of it. Evidently, the divergent oscillatory curve does not enter $I_{0}$ in this case. If $\mathbf{D I}_{0}$ is not constant and its components are only partially in $I_{0}$ then the corresponding solution may or may not converge.

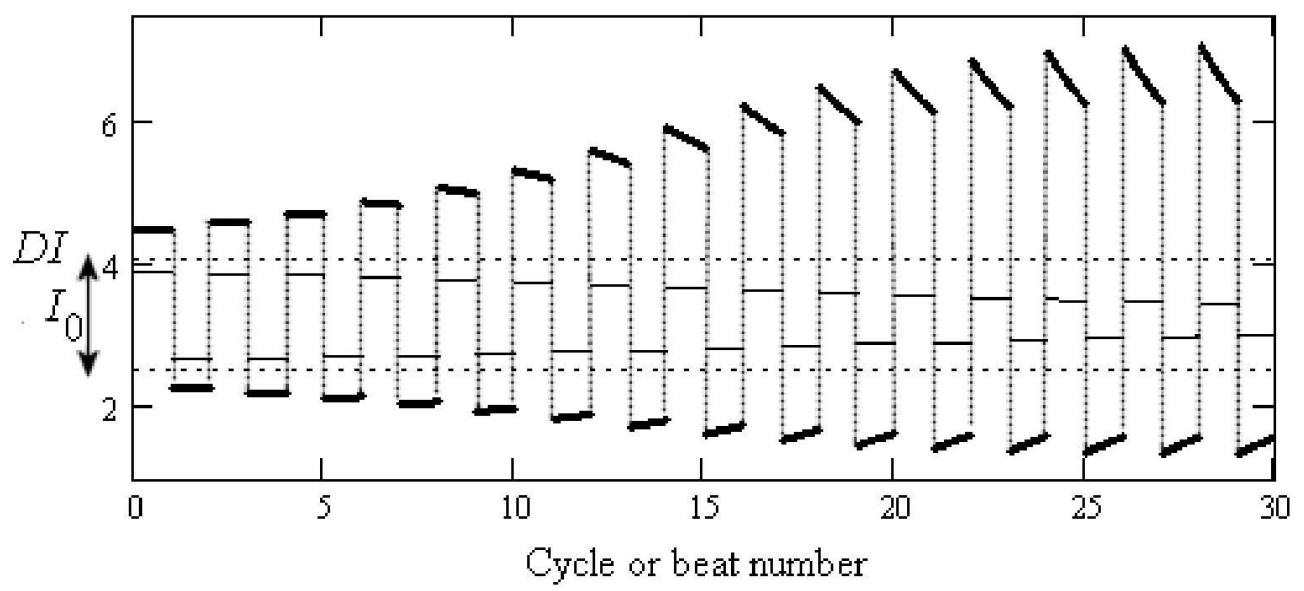

Figure 4: Coexisting stable orbits

FIG.4: This figure shows the qualitatively different outcomes that result from slightly different initial values. With initial values $D I_{0}=[3.9, \ldots, 3.9]$ we obtain the convergent solution shown as the thin, square-shaped 
oscillatory curve, since 3.9 is in $I_{0}$. However, with $D I_{0}=[4.5, \ldots, 4.5]$ we obtain the divergent, thick oscillatory curve. Note that 4.5 is in $I$ but not in $I_{0}$. The horizontal strip between the dotted lines represents $I_{0}$ in this figure.

More complex types of oscillatory behavior (not shown) where the trajectory may pass through $I_{0}$ repeatedly also occur in the above context with different parameter values. In such cases, the occurrence of convergent solutions may again be explained by Theorem 1 if $\mathbf{D I}_{0} \in I_{0}^{m}$ (even with inhomogeneities). However, explaining the stable occurrence of the non-convergent orbits requires further extensions of Theorem 2, or perhaps different methods that are global in nature; local arguments and linearization in $m$ dimensions do not apply since the equilibrium is asymptotically stable within $I_{0}^{m}$.

Remark. (Emergence of bistability) Variations of shapes or changes in the relationship between the CT and APD curves may cause the emergence of bistability by bringing $x^{*}$ close to the region of local flatness for the APD. For illustration, consider Eq.(8) again where we change the parameter $L$ (the length of the loop, a common bifurcation parameter). Note that $x^{*}=\phi^{-1}(L)$ is a strictly increasing function of $L$, where

$$
\phi(x)=\frac{144+6 x-72 e^{-0.5 x}+1.8 e^{-(x-2.5)^{2}}}{1+e^{-4 x}}, \quad x>0
$$

is easily obtained by solving Eq.(11) for $L$ subject to the functions $A, C$ and their parameter values for Fig.3. Thus we can reduce $x^{*}$ by decreasing $L$ and examine of the effect of local APD flatness on bistability as $x^{*}$ passes through the region of relative flatness for the APD curve. To visually demonstrate this effect of $L$ on bistability we plot the two variable curve

$$
F(L, F(L, x))=x, \text { where } F(L, x)=\frac{L}{6}\left[1+e^{-4 x}\right]-24+12 e^{-0.5 x}-p e^{-(x-2.5)^{2}}
$$

for both zero and nonzero (fixed) values of $p$. Such a bifurcation diagram shows the motion of the equilibrium for Eq.(8) as well as the occurrence and development of all period-2 points of the auxiliary map $F$ as $L$ varies. Fig. 5 below shows a comparison between two cases. 

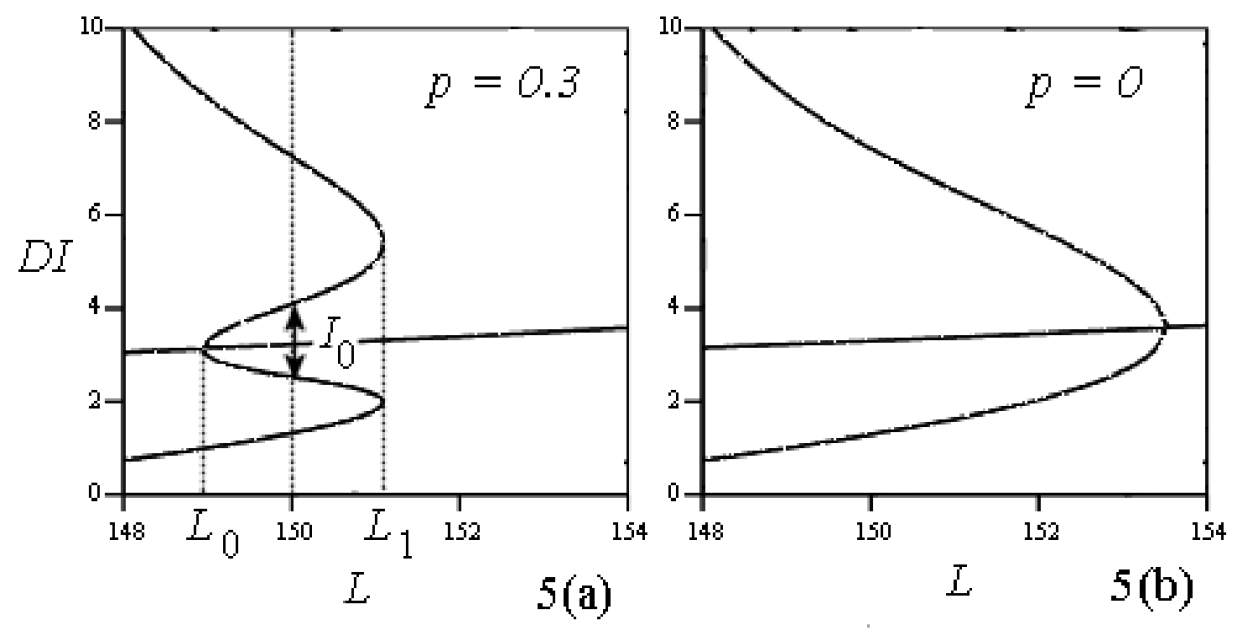

Figure 5: Emergence of bistability

FIG.5: As the length Lof the ring increases, bistable behavior emerges in (a) but not in (b). The occurrence of a tangent (or fold) bifurcation in (a) creates two different period-2 orbits, the inner ones being unstable and enclosing a copy of the interval $I_{0}$ of Fig. 3 between them.

In both of the cases (a) and (b) shown in Fig.5, the nearly horizontal thick curve indicates the path of $x^{*}$ as $L$ changes. The other thick curve in Fig.5(a) where $p \neq 0$ (local flatness exists in APD) clearly shows the emergence of bistability at $L_{1} \approx 151.1$ where a tangent (or fold) bifurcation of the auxiliary map $F$ creates two period-2 orbits (for $F$ ). The two outer curves indicate the stable 2-cycles of $F$ and the inner curves show the unstable 2-cycles which converge to the equilibrium $x^{*}$ at $L_{0} \approx 149$ through a reverse period-doubling (or flip) bifurcation. The interval between the two inner curves exists nontrivially for each $L \in\left(L_{0}, L_{1}\right)$ and gives the interval $I_{0}=I_{0}(L)$ of bistability (by Theorem $1, x^{*}$ is asymptotically stable for all $L \in\left(L_{0}, L_{1}\right)$.) The line segment with arrows in Fig.5(a) indicates the interval $I_{0}$ at the particular value $L=150$; this is just the interval $I_{0}$ that appears in Figures 3 and 4. By contrast, in Fig.5(b) where there is no region of relative flatness for the APD curve, the interval $I_{0}$ does not exist. For some related remarks concerning the occurrence of bistable behavior, see Vinet [25]. Vinet also uses a parameter $p$ by which the APD function can be altered. However, the bifurcation diagrams in [25] are based on variation of $p$ rather than $L$.

\section{Concluding Remarks.}

As indicated above, many models of reentry in a loop of cardiac tissue or more generally, of excitable media have been studied in the literature both in continuous and discrete form. In this paper, we 
refine and extend previous work using new ideas and methods that yield global results. But more work remains to be done, even (and perhaps especially) at the level of pure mathematics. For instance, it is necessary to extend Theorems 1 and 2 so as to cover a broader range of possibilities, or to obtain results that are valid for relevant ranges of various parameters in the more specific equations like (8) that are likely to be obtained through curve fitting of experimental data. It is also necessary to study extensions of these models that use more general types of restitution, e.g., to account for beat to beat memory, latency, etc.

Further, one sees that the persistent oscillation exhibited by the solutions of (6) is generally not complicated in nature (the reasons for the absence of complexity with monotonic APD are easy to discern when $C$ is constant). Can more complex types of persistent oscillations occur, e.g., with higher periods? Can they be possibly chaotic? In certain papers evidence is given that the APD restitution curve $A$ may be non-monotonic; e.g., be unimodal [24] or contain dents [22] at small DI values. In these works it is shown that chaotic behavior and complex bifurcations may occur whether it is for reentry in a loop [22] or for a regularly paced, non-closed strip of tissue [24]. These observations may also be confirmed through numerical simulations of Eq.(8) above with large enough $p$ (not presented here). Such studies indicate the existence of a spatio-temporal form of chaotic behavior that needs further mathematical study and clarification.

\section{REFERENCES}

[1] R.P. Agarwal, Difference Equations and Inequalities: Theory, Methods and Applications, (2nd ed) Marcel-Dekker, New York, 2000.

[2] I. Banville and R.A. Gray, Effects of action potential duration and conduction velocity restitution on alternans and the stability of arrhythmias, J. Cardiovascular Electrophysiol., 13 (2002), pp.1141-1149.

[3] D.R. Chialvo and J. Jalife, On the nonlinear equilibrium of the heart: Locking behavior and chaos in Purkinje fibers, In: Cardiac Electrophysiology: From Cell to Bedside, D.P. Zipes and J. Jalife (Ed.s), Chapter 24, Saunders, Philadelphia, 1990.

[4] P. Collet and J-P. Eckmann, Iterated Maps on the Interval as Dynamical Systems, Birkhauser, Boston, 1980.

[5] M. Courtemanche, L. Glass and J.P. Keener, Instabilities of a propagating pulse in a ring of excitable media, Phys. Rev. Lett., 70 (1993), pp.2182-2185.

[6] M. Courtemanche, J.P. Keener and L. Glass, A delay equation representation of pulse circulation on a ring in excitable media, SIAM J. Appl. Math., 56 (1996), pp.119-142.

[7] M. Courtemanche and A. Vinet, Reentry in excitable media, In: A. Beuter, L. Glass, M.C. Mackey and M.S. Titcombe (Ed.s), Nonlinear Dynamics in Physiology and Medicine, Chapter 7, Springer, New York, 2003.

[8] S.N. Elaydi, An Introduction to Difference Equations, (2nd ed) Springer, New York, 1999.

[9] J.J. Fox, E. Bodenschatz and R.F. Gilmour, Period-doubling instability and memory in cardiac tissue, Phys. Rev. Lett., 89 (2002), pp.8101-8104. 
[10] L.H. Frame and M.B. Simson, Oscillations of conduction, action potential duration and refractoriness, Circulation, 78 (1988), pp.2182-2185.

[11] R.F. Gilmour, N.F. Otani and M.A. Watanabe, Memory and complex dynamics in cardiac Purkinje fibers, Amer. J. Physiol., 272 (4 Pt 2) (1997), pp.H1826-H1832.

[12] J. Guckenheimer and P. Holmes, Nonlinear Oscillations, Dynamical Systems and Bifurctions of Vector Fields, Springer, New York, 1983.

[13] G.M. Hall, S. Bahar and D.J. Gautheir, Prevalence of rate-dependent behaviors in cardiac muscle, Phys. Rev. Lett., 82 (1999), pp.2995-2998.

[14] H. Ito and L. Glass, Theory of reentrant excitation in a ring of cardiac tissue, Physica D, 56 (1992), pp.84-106.

[15] S.S. Kalb, H.M. Dobrovolny, E.G. Tolkacheva, S.F. Idriss, W. Krassowska and D.J. Gauthier, The restitution portrait: A new method for investigating rate-dependent restitution, J. Cardiovascular Electrophysiol., 15 (2004), pp.698-709.

[16] J.P. Keener, Arrhythmias by dimension, In: An Introduction to Mathematical Modeling in Physiology, Cell Biology and Immunology, J. Sneyd (Ed.), pp.57-81, American Mathematical Society, 2002.

[17] W.G. Kelley and A.C. Peterson, Difference Equations: An Introduction with Applications (2nd ed), Harcourt/Academic Press, San Diego, 2001.

[18] V.L. Kocic and G. Ladas, Global Behavior of Nonlinear Difference Equations of Higher Order with Applications, Kluwer, Dordrecht, 1993.

[19] V. Lakshmikantham and D. Trigiante, Theory of Difference Equations: Numerical Methods and Applications (2nd ed), Marcel-Dekker, New York, 2002.

[20] R. Mickens, Difference Equations: Theory and Applications (2nd ed), CRC Press, Boca Raton, 1991.

[21] N.F. Otani and R.F. Gilmour, Memory models for the electrical properties of local cardiac systems, J. Theor. Biol., 187 (1997), pp.409-436.

[22] Z. Qu, J.N. Weiss and A. Garfinkel, Spatiotemporal chaos in a simulated ring of cardiac cells, Phys. Rev. Lett., 78 (1997), pp.1387-1390.

[23] H. Sedaghat, Nonlinear Difference Equations: Theory with Applications to Social Science Models, Kluwer, Dordrecht, 2003.

[24] M.D. Stubna, R.H. Rand and R.F. Gilmour, Analysis of a nonlinear partial difference equation, and its application to cardiac dynamics, J. Difference Equations and Appl., 8 (2002), pp.1147-1169.

[25] A. Vinet, Quasiperiodic circus movement in a loop model of cardiac tissue: Multistability and low dimensional equivalence, Ann. Biomed. Eng., 28 (2000), pp.704-720.

[26] M.A. Watanabe and M.L. Koller, Mathematical analysis of dynamics of cardiac memory and accomodation: Theory and experiment, Am. J. Physiol., 282 (2002), pp.H1534-H1547. 